\title{
Telemedicine in haemophilia during COVID-19 and beyond: a comprehensive review
}

Casey L Li, Anthony KC Chan, Davide Matino, Mihir D Bhatt, Kay Decker, Karen Strike

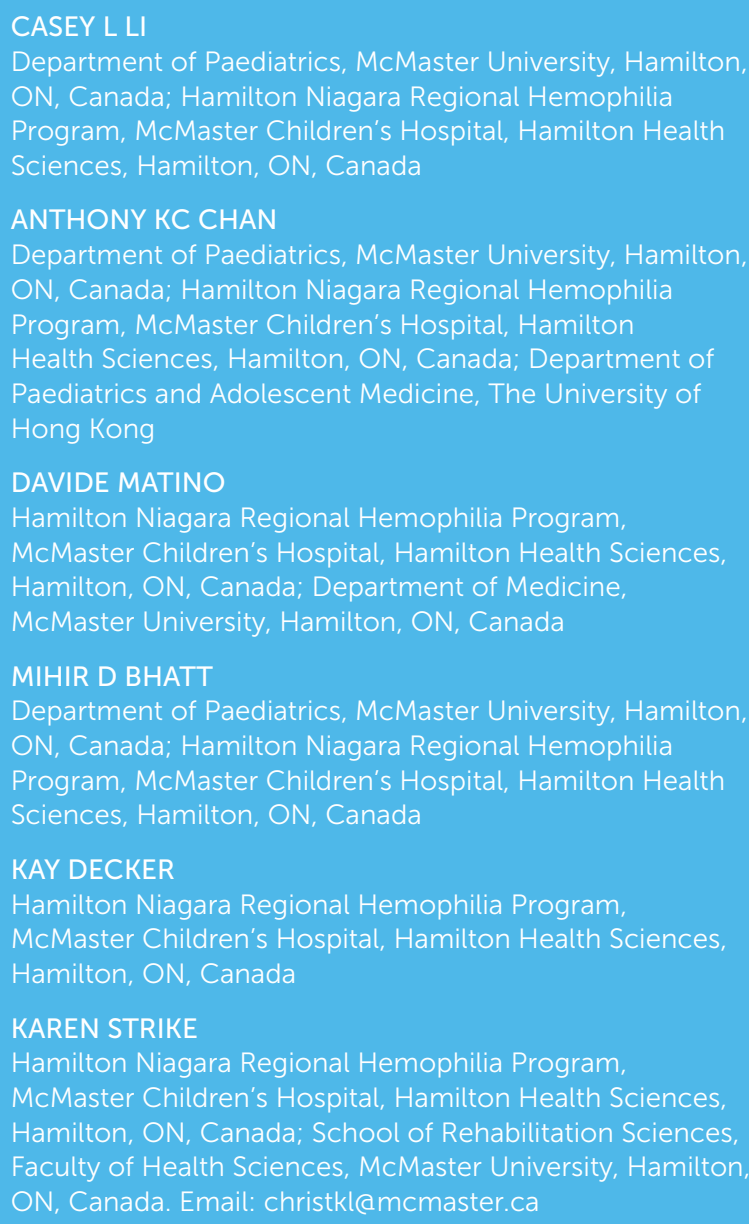

Introduction: Patients with haemophilia require regular assessments and physical examinations. The COVID-19 pandemic has resulted in the rapid adoption of telemedicine to enable virtual consultations and

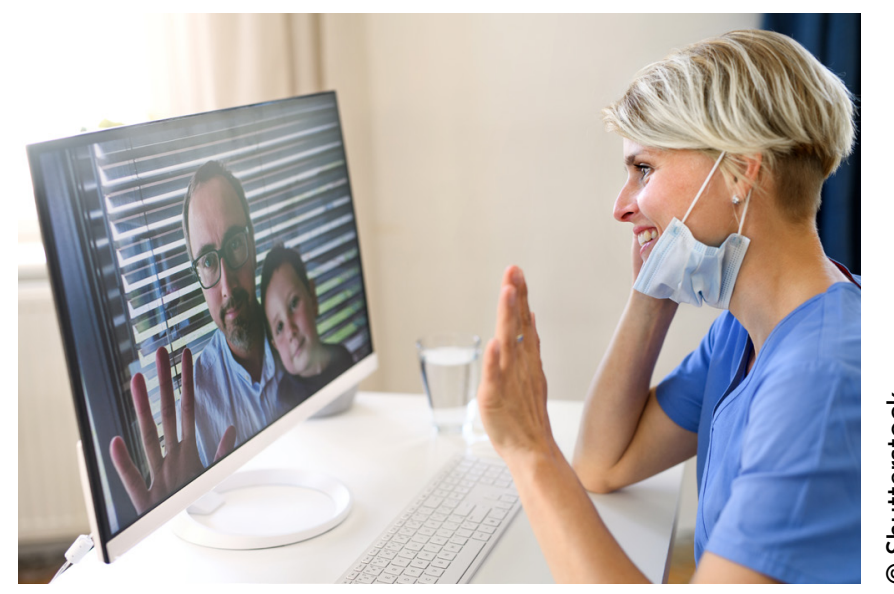

Following its rapid adoption during COVID-19, there has been broad acceptance and recognition of the benefits of telemedicine in haemophilia care. Standardised guidelines on the virtual consultation process and how best to use clinical tools in a virtual setting could further transform practice.

reduce hospital visits. However, the process of virtual consultations is new to many haemophilia clinics. A better understanding of best practices in telemedicine is important to ensure optimal quality of care for patients with haemophilia. Objectives: To summarise the current literature on the use of direct-to-consumer telemedicine for patients with haemophilia and to describe the effectiveness and potential limitations of the technology and methods used. Methods: A comprehensive search was conducted in MEDLINE and EMBASE databases using terms referring to the concepts "haemophilia" AND "telemedicine" and their synonyms. There were no time or language restrictions. Title, abstracts, and full texts were 
screened. Included articles involved telemedicine interventions to facilitate clinical services directly between patients and providers without the use of third-party personnel. The primary outcome was the satisfaction of providers and patients. Secondary outcomes included economic considerations and clinical outcomes. Information was extracted based on study-specific, patient-specific, intervention-specific, and outcome-specific data. Results: Of the 925 articles screened, six were identified and summarised. Three described telemedicine within the context of COVID-19. Technologies used included telephone calls, videoconferencing, text messaging, and email. All studies involved a multidisciplinary team. Telemedicine in haemophilia care was found to positively impact the patient experience. Providers were satisfied with telemedicine. It was also suggested to be economically beneficial and positively impacted patient outcomes. However, none of the articles reported on how telemedicine was specifically used to perform assessments during the virtual consultation process. Conclusions: There is preliminary evidence that telemedicine may be beneficial in haemophilia care. Overall, patients and providers reported high satisfaction with the usage of direct-to-consumer telemedicine. This positive reception warrants improvements in standardisation of reporting and quality of study design to better assess its clinical and economic impact. Developing a standard guideline for virtual consultations would support healthcare practitioners in how to best incorporate telemedicine to improve quality of care.

Keywords: Telemedicine, haemophilia, COVID-19, consultation, technology

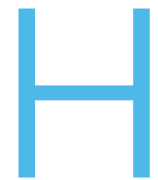

aemophilia is a family of bleeding disorders resulting from the deficiency of coagulation factors ${ }^{[1]}$. The spontaneous or inherited pathogenic gene mutations are typically $\mathrm{X}$-linked, and most commonly affect coagulation factors VIII and IX in haemophilia A and $B$, respectively ${ }^{[1]}$. As a rare genetic condition, the prevalence of haemophilia is estimated to be $1,125,000$ individuals globally, with haemophilia A accounting for approximately $80-85 \%$ of cases, and haemophilia $B$ accounting for $15-20 \%$ of cases ${ }^{[1,2]}$

Patients with deficient or absent coagulation factors typically present with symptoms associated with impaired secondary haemostasis. This includes prolonged post-traumatic and post-surgical bleeding, in addition to deep bleeding in the gastrointestinal tract, brain, muscles, and joints ${ }^{[1]}$. Particularly, joint bleeds are prevalent in paediatric patients, and often occur spontaneously. As a result, joint bleeding may be easily missed, which can lead to long-term consequences in joint mobility ${ }^{[1]}$. In this context, regular assessment and consultations by healthcare providers (HCPs), including nurses, physicians, social workers, and physiotherapists, are important in improving patient outcomes.

Despite the essential role of regular visits to monitor and treat haemophilia, optimal patient care continues to be constrained by limited accessibility, especially in rural areas ${ }^{[3]}$. Given the low prevalence of haemophilia, patients often have to travel to larger, urban treatment centres to attend consultations with their comprehensive care team. Reported benefits of telemedicine include increased convenience in accessing services ${ }^{[4]}$, increased patient satisfaction ${ }^{[4,5]}$, improved clinical outcomes ${ }^{[6]}$, and long-term cost savings to patients and the healthcare system by improving efficiency ${ }^{[4-7]}$. Alongside recent technological advances, these benefits have led to telehealth becoming increasing popular as a potential method for improving healthcare delivery.

Telehealth and telemedicine are terms that are often used interchangeably but by definition they describe different concepts. Telehealth involves the use of technology in both remote and non-remote settings to deliver healthcare to patients who may experience healthcare inaccessibility due to social, cultural or geographic barriers ${ }^{[8]}$. Telemedicine, on the other hand, is more specific and involves the remote provision of clinical services ${ }^{[8]}$. This paper focuses on the use of telemedicine due to the need for remote clinical service delivery during the coronavirus pandemic 2019 (COVID-19). Prior to the pandemic, telemedicine was developed primarily to provide care to remote areas.

Historically, the healthcare system has been slow to embrace telemedicine due to concerns around patient confidentiality, data security, economic barriers, upfront costs, difficulties using technologies, and patient preferences for face-to-face interactions ${ }^{[5]}$. However, due to the COVID-19 pandemic health teams globally have been quick to adapt to these methods.

COVID-19 was first reported in December 2019 in Wuhan, Hubei Province, China ${ }^{[9]}$ and was recognised by the World Health Organization as a "pneumonia of unknown etiology" on 5 January $2020{ }^{[10]}$. COVID-19 has affected hospital capacity and resulted in a shortage of hospital resources globally due to pandemic-related emergency surges ${ }^{[11]}$. The use of telemedicine has been previously described as a method to respond 
to disasters ${ }^{[12]}$. The emergence of COVID-19 has resulted in a movement towards reducing face-toface provider-patient contact in order to decrease the risk of transmission, resulting in an increasing demand to incorporate digital technology in delivering clinical services.

At the beginning of the COVID-19 pandemic, many healthcare sites around the world temporarily postponed non-essential medical visits and began to implement telemedicine. As telemedicine remains novel for many HCPs, it is important to evaluate best practices in virtual consultations. Due to the nature of the condition, the physical examination process has been an important aspect of in-person visits in haemophilia care. However, performing these physical exams virtually presents challenges. Gathering and disseminating the experiences of healthcare teams and outlining methodologies and insights can help inform practice in performing virtual consultations. This can enable HCPs to learn and prepare for similar future challenges and help telemedicine to become an effective alternative method of providing care.

During COVID-19, it became important to discuss direct-to-consumer telemedicine, whereby telemedicine is delivered directly between patients and their providers with no intermediary body (e.g. another HCP). For the purposes of this review, we include both synchronous and asynchronous forms of direct-to-consumer telemedicine. 'Synchronous' describes real-time telemedicine applications, such as videoconferencing. 'Asynchronous', also known as a store-and-forward system, describes information relayed over a period of time and may not be immediate, such as photos uploaded through email.

The purpose of this review was to summarise the current literature available on telemedicine technology and the methods that facilitate direct-toconsumer delivery of clinical services for patients with haemophilia. A secondary purpose was to report on the effectiveness of the technology and methods used, including cost-effectiveness and patient and provider satisfaction. We aim to provide recommendations based on published data and experiences to help guide the telemedicine consultation process for HCPs who work with people with haemophilia.

\section{METHODS}

\section{Search strategy}

A literature search of articles published in peerreviewed journals up to 24 October 2020 was conducted by a reviewer (CL) through MEDLINE, Embase, and PubMed databases, using the search terms "telemedicine", "hemophilia", and their synonyms. The search strategies are included in the Appendix. Titles and abstracts were screened for relevance. If the reviewer was unsure whether an article met the inclusion criteria, a second reviewer was consulted. The full texts of all relevant abstracts were then screened for inclusion. All full text articles that addressed telemedicine used directly between consumer/patients and their providers in the context of haemophilia were included. The reference lists of included articles were hand searched to identify any additional articles that were not captured by the database search.

\section{Study selection}

All included articles involved telemedicine interventions to facilitate clinical services directly between patients with haemophilia and providers without the use of a third party (e.g. another HCP). Articles were excluded if a third party was used. Studies involving virtual interventions that were not used for the purposes of consultation were excluded. Review articles, abstracts, and conference proceedings were excluded, although those identified as relevant were also hand searched for additional articles. All other study designs were included, such as observational studies, retrospective and prospective cohort studies, case-control studies, case reports, and cross-sectional studies. There were no date or language restrictions in order to capture all available papers. The eligible dates from the databases are included in the Appendix (Search strategies).

\section{Data collection}

Data was extracted by CL. Data collection was based on study-specific, patient-specific, intervention-specific, and outcome-specific data. Study-specific data included the study authors, article title, journal, and country. Patient-specific data included the severity of haemophilia, ages of the patients, and virtual medium used. Intervention-specific data included the virtual medium used and the healthcare team's disciplines. Any outcome-specific data reported was collected, including patient satisfaction, provider satisfaction, patient recovery, prevention of hospital visits, patient attendance rates, clinical outcomes, and economic considerations.

A flow diagram illustrating the article search and selection process is shown in Figure 1. 
Figure 1. Flow Diagram

Diagram illustrating the flow of information through the different phases of the review process for identifying current literature available on telemedicine technology and the methods that facilitate direct-to-consumer delivery of clinical services for patients with haemophilia. The process follows that outlined in the PRISMA Statement $2009^{[27] .}$

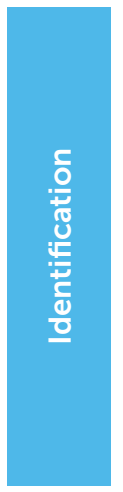

Records identified through database searching $(n=925)$

PubMed $(n=214)$

Embase $(n=557)$

MEDLINE $(n=154)$

Additional records identified through other sources $(n=0)$

Records after duplicates removed

$(n=666)$
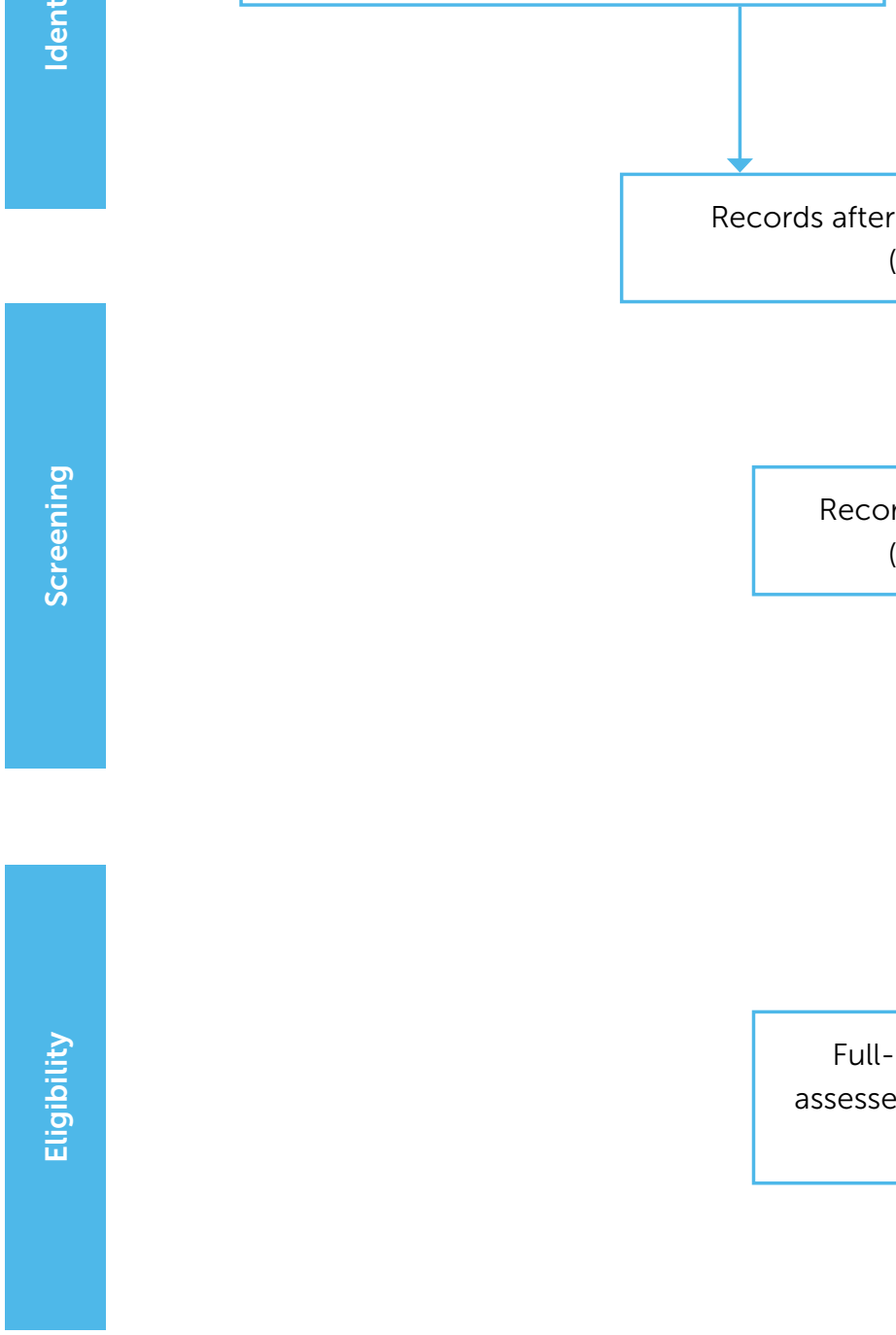

Records excluded $(n=613)$
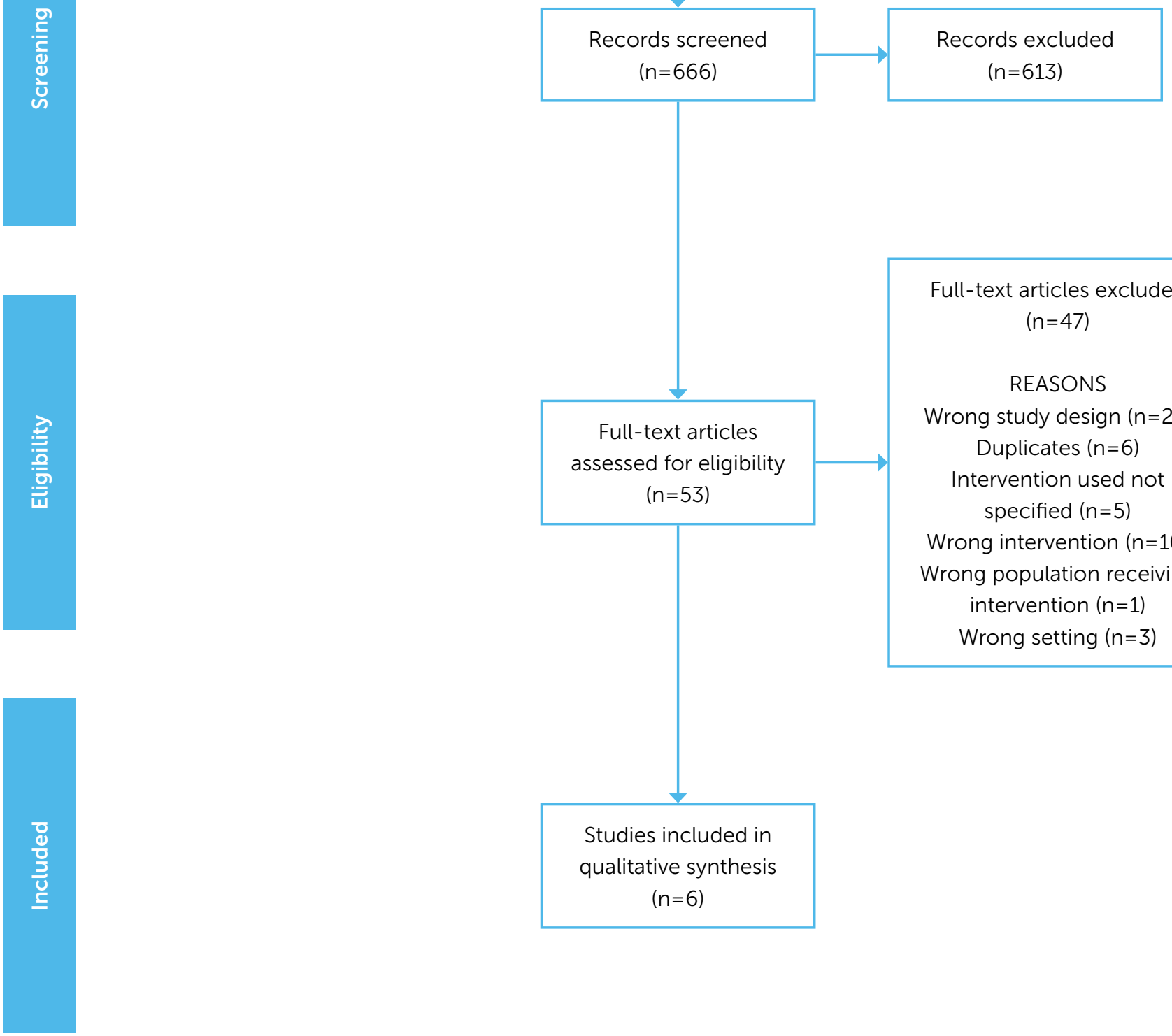

Full-text articles assessed for eligibility $(n=53)$

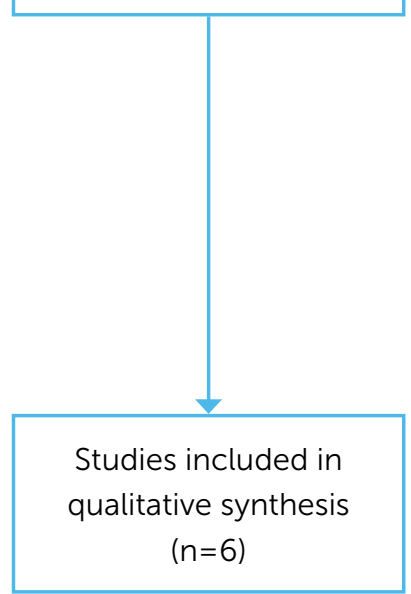

Full-text articles excluded $(n=47)$

\section{REASONS}

Wrong study design $(n=22)$

Duplicates $(n=6)$

Intervention used not specified $(n=5)$

Wrong intervention $(n=10)$

Wrong population receiving intervention $(n=1)$

Wrong setting $(n=3)$ 


\section{RESULTS}

Of 925 search results yielded, 259 duplicates were removed, and the 666 remaining articles were screened for inclusion. Hand searches did not reveal any additional relevant articles. Six articles describing the use of telemedicine in haemophilia between consumers/patients and HCPs published between 2015 and 2020 were identified (Table 1). These used multiple study designs including crosssectional surveys $(n=3)^{[13-15]}$, a retrospective cohort study $(n=1)^{[16]}$, a cross-sectional population-based study $(n=1)^{[17]}$, and a case-report $(n=1)^{[18]}$.

The included articles originated from six different countries: USA ${ }^{[13]}$, Ireland ${ }^{[14]}$, Germany ${ }^{[16]}$, China ${ }^{[17]}$, The Netherlands ${ }^{[15]}$, and Spain ${ }^{[18]}$. Half of the publications described telemedicine use in haemophilia during the COVID-19 pandemic ${ }^{[14,17,18]}$. All patients in the studies were between the ages of 6 months and 91 years old. In total 2,237 patients were included in the six studies.

\section{Methods of direct-to-consumer telemedicine}

Various technologies were used during the telemedicine consultation process, with some studies using more than one. Five described the use of telephone calls [13-17], two involved the use of text/SMS messaging ${ }^{[16,17]}$, two used email ${ }^{[13,18]}$, and five used videoconferencing ${ }^{[13-15,17,18]}$. Five articles described the use of photographs through text, email, or a mobile application as part of the consultation process ${ }^{[13,15-18]}$.

All six articles described the use of telemedicine involving a multidisciplinary team including different healthcare professionals ${ }^{[13-18]}$. However, it was unclear in two articles which healthcare professionals were involved in the "hemophilia treatment center" ${ }^{[16]}$ or who the "healthcare providers" were ${ }^{[13]}$.

Some articles mentioned the assessments conducted, including joint range of motion assessment ${ }^{[13,14,18]}$, muscle strength ${ }^{[18]}$, and gait assessment ${ }^{[14]}$. However, none of the articles described how the assessments were performed or provided details on instruments used, questions asked, and instructions provided to patients to perform these assessments accurately.

\section{Satisfaction with telemedicine}

Patient satisfaction was described in three studies, each of which measured this using study-specific surveys with different questions ${ }^{[13-15]}$. None of the studies used surveys that were validated by sources external to the study. Participants of all three studies described both positive and negative experiences with telemedicine ${ }^{[19]}$.
Patient experiences using teleconsultation were largely positive ${ }^{[13-15]}$. The most commonly reported benefits of telemedicine contributing to patient satisfaction included ease of use ${ }^{[13-15]}$, helpfulness in patient-provider communication ${ }^{[14,15]}$, and reduction in travel time and cost ${ }^{[13,14]}$. Adolescents in one study commented that telemedicine improved feelings of independence and agency in caring for themselves ${ }^{[13]}$. However, all three studies reported equipment, internet, and connection failures as a source of frustration for patients ${ }^{[13-15]}$. Limitations with available devices for teleconsultation ${ }^{[15]}$ and accessibility issues such as language and visual impairments ${ }^{[14]}$ affected the patient experience.

Provider satisfaction was described in four studies ${ }^{[13-15,18]}$. Three used study-specific surveys but provided little detail on their content, and one (a case report) did not clarify how provider satisfaction was ascertained. In one study with 21 respondents, $90 \%$ and $86 \%$ of HCPs felt confident using telephone and video consultation, respectively ${ }^{[14]}$. Here, 79\% of HCPs responded that they would likely continue teleconsultation as routine care, and $88 \%$ would recommend telemedicine to their colleagues ${ }^{[14]}$. Among physiotherapists, $94 \%$ of survey respondents were satisfied or very satisfied with their experience of videoconferencing consultations ${ }^{[14]}$. Gait assessment was satisfactory but joint range of motion assessment, despite being possible, may require evaluation inperson ${ }^{[14]}$. HCPs in three studies believed that using teleconsultation helped prevent unnecessary patient visits to treatment centres ${ }^{[13-15]}$. Interestingly, it was identified in a study with four respondents that the use of videoconferencing was not always necessary, as some providers felt that similar information could be conveyed with the use of telephones ${ }^{[13]}$. A case report described that providers in Haematology, Orthopaedics, and Rehabilitation Departments found telemedicine to be useful for a patient with severe haemophilia A after undergoing orthopaedic surgery ${ }^{[18]}$. One study reported limitations of telemedicine described by HCPs. Technical difficulties were found to occur in $38 \%$ and $71 \%$ of telephone and video consultations, respectively; $67 \%$ of HCPs reported not feeling adequately prepared in providing video consultations; and $71 \%$ of HCPs were interested in receiving further education in telemedicine ${ }^{[14]}$.

\section{Cost-effectiveness of telemedicine}

Cost-effectiveness of telemedicine was described in four studies ${ }^{[14-17]}$, and three studies demonstrated that using telemedicine decreased the need for hospital 
visits $^{[14,15,17]}$. One study reported that the introduction of teleconsultation reduced clinic non-attendance rates by $60 \%{ }^{[14]}$. In one study the HCP was able to assess bleeding severity adequately in eight out of nine video consultations, which prevented the need for a hospital visit ${ }^{[15]}$. This was also demonstrated in a study conducted in Ireland, in which $49 \%$ of patients who utilised teleconferencing lived a significant distance from their haemophilia centre and would have had to travel up to three hours to attend it ${ }^{[14]}$. In another study bleeding frequency was reduced after the usage of an electronic diary with videoconferencing capabilities ${ }^{[16]}$.

\section{COVID-19 and telemedicine}

It is important to mention that, at the time of writing, the COVID-19 pandemic continues to affect healthcare delivery globally. Our search yielded three articles on telemedicine's specific application to haemophilia in the context of COVID-19 ${ }^{[14,17,18]}$, with one demonstrating its implementation in Wuhan, the epicentre of the pandemic, in addition to Tianjin ${ }^{[17]}$. In both cities, teleconsultations were implemented in most hospitals from early February 2020, when in-person assessments were not available. This enabled HCPs to guide factor administration, adjust dosages, handle mild bleeding and prescribe coagulation factors, while reducing visits to local hospitals during the lockdown. For those without access to technology or the ability to conduct teleconsultations, home visit services were used as an alternative to in-person hospital visits when possible.

The use of telemedicine in COVID-19 was also described in a case report from Spain, where the pandemic accelerated the implementation of telemedicine services for haemophilia ${ }^{[18]}$. A patient with severe haemophilia A underwent a major orthopaedic surgery and was followed up through telemedicine. This minimised the risk of COVID-19 infection from hospital visits, and enabled HCPs to monitor the patient throughout their postoperative management and rehabilitation.

In another cross-sectional survey conducted in Ireland, COVID-19 resulted in a drop in clinical consultations from 252 during March 2019 to 94 in March $2020{ }^{[14]}$. The implementation of telemedicine in March 2020 enabled the clinic to recover to 230 consultations in April $2020{ }^{[14]}$. A 52\% increase in medical consultations ( $n=407$ ) was seen in May 2020 compared to the same month in the year prior to the COVID-19 pandemic $(n=268)$, exceeding the clinic's original capacity. Furthermore, a $60 \%$ reduction in nonattendance was seen with the use of telehealth. Overall, telemedicine has been demonstrated as an effective intervention to mitigate effects of COVID-19 on clinical operations.

\section{DISCUSSION}

Telemedicine was found to be a positive experience for most users before and during COVID-19 and presents as a cost-effective method in delivering clinical consultations. Owing to the rapid adoption of teleconsultation in various healthcare systems during COVID-19, there is increased literature surrounding its use and efficacy in patient care. The six studies identified in our review were from multiple countries, suggesting that the findings could apply to other countries. Positive patient experiences of telemedicine included ease of use, better provider-patient communication, and reduced travel time and cost. A feeling of independence reported by adolescents is particularly relevant: many in this age group already use digital devices regularly and this may be a familiar form of communication.

Besides improving patient satisfaction, telemedicine was reported to provide a cost-saving alternative to traditional, in-person visits that resulted in positive clinical outcomes. For example, it was found that there were increased follow-up and reduced non-attendance rates. This may be due to the increased convenience of virtual visits, which may lead to increased treatment compliance and the ability for HCPs to monitor patients closely. This, in turn, may reduce bleeding frequency and limit costs associated with risk of later complications. It was also reported that telemedicine reduced the need for patients to visit haemophilia treatment centres, which can result in decreases in travel time and associated costs, such as hospital parking or transport costs.

However, drawbacks of telemedicine have also been reported, including technical difficulties such as equipment and connection failure, poor accessibility, and limited devices available for patients. Particularly, the inability to perform a direct physical exam remains one of the most pressing issues in telemedicine for haemophilia care. It is therefore important to identify and standardise any instruments or methods used to allow patients to conduct accurate measurements when performed virtually. None of the articles in our review clearly outlined the specific methods of the virtual consultation process, such as how specific clinical exams like the joint exam were performed. Therefore, although there are reported benefits to telemedicine in haemophilia care, no methods were reported to assist other professionals to replicate these 
practices. There is an overall need for validated clinical tests that can be employed during virtual consultations.

Authors of the included studies have drawn similar conclusions to earlier published work. Benefits of telemedicine in the context of haemophilia have been previously discussed in Kulkarni's narrative review and include improved access to care, decreased costs, and improved adherence ${ }^{[20]}$. However, this review was published before COVID-19 and there have been more findings since its publication, and the findings of the current review are not only specific to haemophilia care. The acceptance of telemedicine by patients and providers has been generally positive, enabling reduced travel and waiting times, increased accessibility, increased compliance, and ease of use ${ }^{[21]}$. However, similar to our findings, previous studies have highlighted barriers including technical difficulties, reimbursement issues, and patient preference for face-to-face interactions ${ }^{[21]}$.

Many incoming technologies are currently being studied for their potential use in telemedicine. One prominent example is with range of motion (ROM) assessments. ROM is typically measured in person using a goniometer to assess joint health and reduced loss of functionality due to bleeding; however, remote methods of determining ROM are useful for virtual consultation purposes. Kinect V2 is a sensor that detects motion. It has been proposed as a tool that may assist in measuring ROM virtually and has been tested in some studies as a method for monitoring elbow ROM in people with haemophilia ${ }^{\text {[22]. HemoKinect }}$ software utilises the Kinect V2 system to evaluate body movements and can send movement reports physiotherapists remotely. Compared to a standard goniometer in measuring elbow ROM, Kinect V2 was demonstrated to report similar measurements, with no statistically significant differences except for the extension angle ${ }^{[22]}$. This is an example of one of many new technologies that may provide an alternative to standard goniometry, with future applications in telemedicine. However, it is important for researchers and clinicians to further study these applications to ensure their applicability in virtual care.

There are many other considerations when implementing new technologies for telemedicine, including data security and patient confidentiality ${ }^{[21,23]}$. For example, it may be difficult to accurately identify individuals based on telephone calls, as this is reliant on the correct identification of the sound of an individual's voice. Ensuring equity and inclusion of services is a key consideration: internet accessibility is not universal and broadband connections are not always available and some individuals may not be comfortable with appearing in front of a screen ${ }^{[21,23]}$. Patients may have visual impairments, language barriers, or a preference for face-to-face interactions. The ageing population may likewise have difficulties using electronic devices and individuals may be resistant to changing current practices. These are all barriers to accessing haemophilia telemedicine, and it is important that robust processes are developed that cater to the populations that experience them.

Despite this, the advent of technological advancements and improved network bandwidth capabilities, means that telemedicine can not only increase healthcare accessibility, but can reduce costs at both an individual and systemic level ${ }^{[7]}$. This is relevant to chronic conditions, where telemedicine interventions such as preventative home monitoring and teleconsultations have previously been shown to reduce hospital admission rates, length of stay, and overall costs, while delivering effective patient outcomes ${ }^{[24-26]}$. For people with haemophilia, time associated with travelling to treatment centres, transportation costs, and inconvenient clinic hours represent significant barriers to effective management of their condition ${ }^{[3]}$.

There is currently limited information regarding specific methods used in the virtual consultation process. The importance of knowledge dissemination combined with the lack of specific details around these methods in the literature encourages a call to action to create a standardised guideline for implementing telemedicine for people with haemophilia. It also becomes important to report the specific methods of telemedicine used so that other centres can better compare and assess the clinical and economic impact. These future processes can assist healthcare professionals in determining how to best incorporate telemedicine to improve patient experience and overall care.

\section{Limitations}

There are limitations to this review. The telemedicine interventions and technologies used in the different countries and hospital centres included in the studies we identified were not clearly outlined and may not have been the same. Due to potential discrepancies in intervention methods, the outcomes of each study may not be comparable to other studies. Additionally, the included studies did not use standardised outcome measurements, meaning that while they may have measured similar concepts, they may not 
have measured the same outcome. For example, where questionnaires were used, the questions and methodology of assessment varied. Finally, telemedicine is rapidly evolving and advancing due to the COVID-19 pandemic, and any outcomes described in published literature may not represent the most recent technological advances.

\section{CONCLUSION}

There is some literature to support the use of direct-toconsumer telemedicine for people with haemophilia. The evidence found in the current review highlights the usefulness of telemedicine, particularly for patients who live at a distance to their haemophilia treatment centre. It may be possible to provide telemedicine consultations in lieu of in-person haemophilia treatment centre clinic visits. However, there is no literature to discuss the effectiveness of specific methods for virtual consultations and it is important that HCPs better document the methods they use to serve this patient population. The COVID-19 pandemic has called for a response to finding innovative telemedicine solutions. Although the implementation of telemedicine has been rapid and by circumstance, there has been broad acceptance of new consultation methods with potential future benefits. With the shift in attitude towards remote consultations between patients and HCPs, it becomes important to measure and understand how effective teleconsultations are within haemophilia care and how consultations are taking place. Telemedicine will continue to be used after the pandemic and there is a need to disseminate information on the processes of using clinical tools in a virtual setting to transform practice.

\section{ACKNOWLEDGEMENTS}

The authors have advised no interests that might be perceived as posing a conflict or bias.

Anthony Chan holds the McMaster Children's Hospital/Hamilton Health Sciences Foundation Chair in Pediatric Thrombosis and Hemostasis

This paper does not contain any studies involving human participants or animals performed by any of the authors.

\section{ORCID}

Casey L Li (iD https://orcid.org/0000-0001-5747-7721 Anthony KC Chan (iD https://orcid.org/0000-0003-1551-3995 Davide Matino (iD) https://orcid.org/0000-0002-9902-4074 Mihir Bhatt (iD) https://orcid.org/0000-0003-3233-4067 Kay Decker (D) https://orcid.org/0000-0002-6847-3192 Karen Strike (D) https://orcid.org/0000-0002-5598-2051

\section{REFERENCES}

1. Srivastava A, Santagostino E, Dougall A, Kitchen S, Sutherland M, Pipe SW, et al. WFH Guidelines for the Management of Hemophilia, 3rd edition. Haemophilia 2020; 26(S6): 1-158. doi: 10.1111/hae.14046.

2. Iorio A, Stonebraker JS, Chambost $\mathrm{H}$, et al. Establishing the prevalence and prevalence at birth of hemophilia in males: a meta-analytic approach using national registries. Ann Intern Med 2019; 171(8): 540-6. doi: 10.7326/M19-1208.

3. Saxena K. Barriers and perceived limitations to early treatment of hemophilia. J Blood Med 2013; 4: 49-56. doi: 10.2147/JBM. S43734.

4. Davis P, Howard R, Brockway P. An evaluation of telehealth in the provision of rheumatologic consults to a remote area. J Rheumatol 2001; 28: 1910-3.

5. Pariser P, Pham T-NT, Brown JB, Stewart M, Charles J. Connecting people with multimorbidity to interprofessional teams using telemedicine. Ann Fam Med 2019; 17 (Suppl 1): S57-S62. doi: 10.1370/afm.2379.

6. Rosenfeld BA, Dorman T, Breslow MJ, et al. Intensive care unit telemedicine: Alternate paradigm for providing continuous intensivist care. Crit Care Med 2000; 28(12): 3925-31. doi: 10.1097/00003246-200012000-00034.

7. Dorsey ER, Topol EJ. State of telehealth. N Engl J Med 2016; 375(2): 154-61. doi: 10.1056/NEJMra1601705.

8. Stenberg PL. Rural Individuals' Telehealth Practices: An Overview. Economic Information Bulletin no. 199. US Department of Agriculture, Economic Research Service, November 2018. Available from https://www.ers.usda.gov/publications/pubdetails/?pubid=90529 (accessed 26 August 2021).

9. Zhu N, Zhang D, Wang W, et al. A novel coronavirus from patients with pneumonia in China, 2019. N Engl J Med 2020; 382(8): 727-33. doi: 10.1056/NEJMoa2001017.

10. World Health Organization. COVID-19 - China. Disease outbreak news: Pneumonia of unknown cause - China. 5 January 2020. Available from https://www.who.int/ emergencies/disease-outbreak-news/item/2020-DON229 (accessed 26 August 2021).

11. Weissman GE, Crane-Droesch A, Chivers C, et al. Locally informed simulation to predict hospital capacity needs during the COVID-19 pandemic. Ann Intern Med 2020: M20-1260. doi: 10.7326/M20-1260.

12. Lurie N, Carr BG. The role of telehealth in the medical response to disasters. JAMA Intern Med 2018; 178(6): 745-6. doi: 10.1001/jamainternmed.2018.1314.

13. Jacobson K, Hooke MC. Telehealth videoconferencing for children with hemophilia and their families: a clinical project. J Pediatr Oncol Nurs 2016; 33(4): 282-8. doi: 10.1177/1043454215607340.

14. O'Donovan M, Buckley C, Benson J, et al. Telehealth for delivery of haemophilia comprehensive care during the COVID-19 pandemic. Haemophilia 2020; 26(6): 984-90. doi: 10.1111/hae.14156.

15. Ludikhuize L, Jansen ME, Hooimeijer HL, de Bont ESJM, Tamminga RYJ. Feasibility of video consultations in case of acute complications in children with haemophilia. Haemophilia 2016; 22(6): e567-e70. doi: 10.1111/hae.13109.

16. Mondorf W, Eichler $\mathrm{H}$, Fischer $\mathrm{R}$, et al. Smart Medication ${ }^{\mathrm{TM}}$, an electronic diary for surveillance of haemophilia home care and 
optimization of resource distribution. Hamostaseologie 2019; 39(04): 339-46. doi: 10.1055/s-0038-1675575.

17. Zhang A, Liu W, Poon M-C, et al. Management of haemophilia patients in the COVID-19 pandemic: Experience in Wuhan and Tianjin, two differently affected cities in China. Haemophilia 2020; 26(6): 1031-7. doi: 10.1111/hae.14108.

18. Álvarez-Román MT, De la Corte-Rodríguez H, RodríguezMerchán EC, et al. COVID-19 and telemedicine in haemophilia in a patient with severe haemophilia a and orthopaedic surgery. Haemophilia 2020 Nov 3. doi: 10.1111/hae.14087. [Epub ahead of print]

19. Kruse CS, Krowski N, Rodriguez B, Tran L, Vela J, Brooks $M$. Telehealth and patient satisfaction: a systematic review and narrative analysis. BMJ Open 2017; 7(8): e016242. doi: 10/1136/bmjopen-2017-016242.

20. Kulkarni R. Use of telehealth in the delivery of comprehensive care for patients with haemophilia and other inherited bleeding disorders. Haemophilia 2018; 24(1): 33-42. doi: 10.1111/hae.13364

21. Almathami HKY, Win KT, Vlahu-Gjorgievska E. Barriers and facilitators that influence telemedicine-based, real-time, online consultation at patients' homes: systematic literature review. J Med Internet Res 2020; 22(2): e16407. doi: 10.2196/16407.

22. Mateo F, Carrasco JJ, Aguilar-Rodríguez M, et al. Assessment of Kinect V2 for elbow range of motion estimation in people with haemophilia using an angle correction model. Haemophilia 2019; 25(3): e165-e73. doi: 10.1111/hae.13744.
23. Scott Kruse C, Karem P, Shifflett K, Vegi L, Ravi K, Brooks M. Evaluating barriers to adopting telemedicine worldwide: A systematic review. J Telemed Telecare 2018; 24(1): 4-12. doi: 10.1177/1357633X16674087.

24. Udsen FW, Hejlesen O, Ehlers LH. A systematic review of the cost and cost-effectiveness of telehealth for patients suffering from chronic obstructive pulmonary disease. J Telemed Telecare 2014; 20(4): 212-20. doi: 10.1177/1357633X14533896

25. Dinesen B, Haesum LKE, Soerensen N, et al. Using preventive home monitoring to reduce hospital admission rates and reduce costs: a case study of telehealth among chronic obstructive pulmonary disease patients. J Telemed Telecare 2012; 18(4): 221-5. doi: 10.1258/jtt.2012.110704.

26. Gellis ZD, Kenaley B, McGinty J, Bardelli E, Davitt J, Ten Have T. Outcomes of a telehealth intervention for homebound older adults with heart or chronic respiratory failure: A randomized controlled trial. Gerontologist 2012; 52(4): 541-52. doi: 10.1093/geront/gnr134.

27. Moher D, Liberati A, Tetzlaff J, Altman DG, PRISMA Group. Preferred reporting items for systematic reviews and metaanalyses: the PRISMA statement. PLoS Med 2009; 6(7): e1000097. doi: 10.1371/journal.pmed.10000097.

HOW TO CITE THIS ARTICLE:

Li CL, Chan AKC, Matino D, Bhatt MD, Decker K, Strike

K. Telemedicine in haemophilia during COVID-19 and beyond: a comprehensive review. J Haem Pract 2021; 8(1): 98-110. https://doi.org.10.2478/jhp-2021-0011.

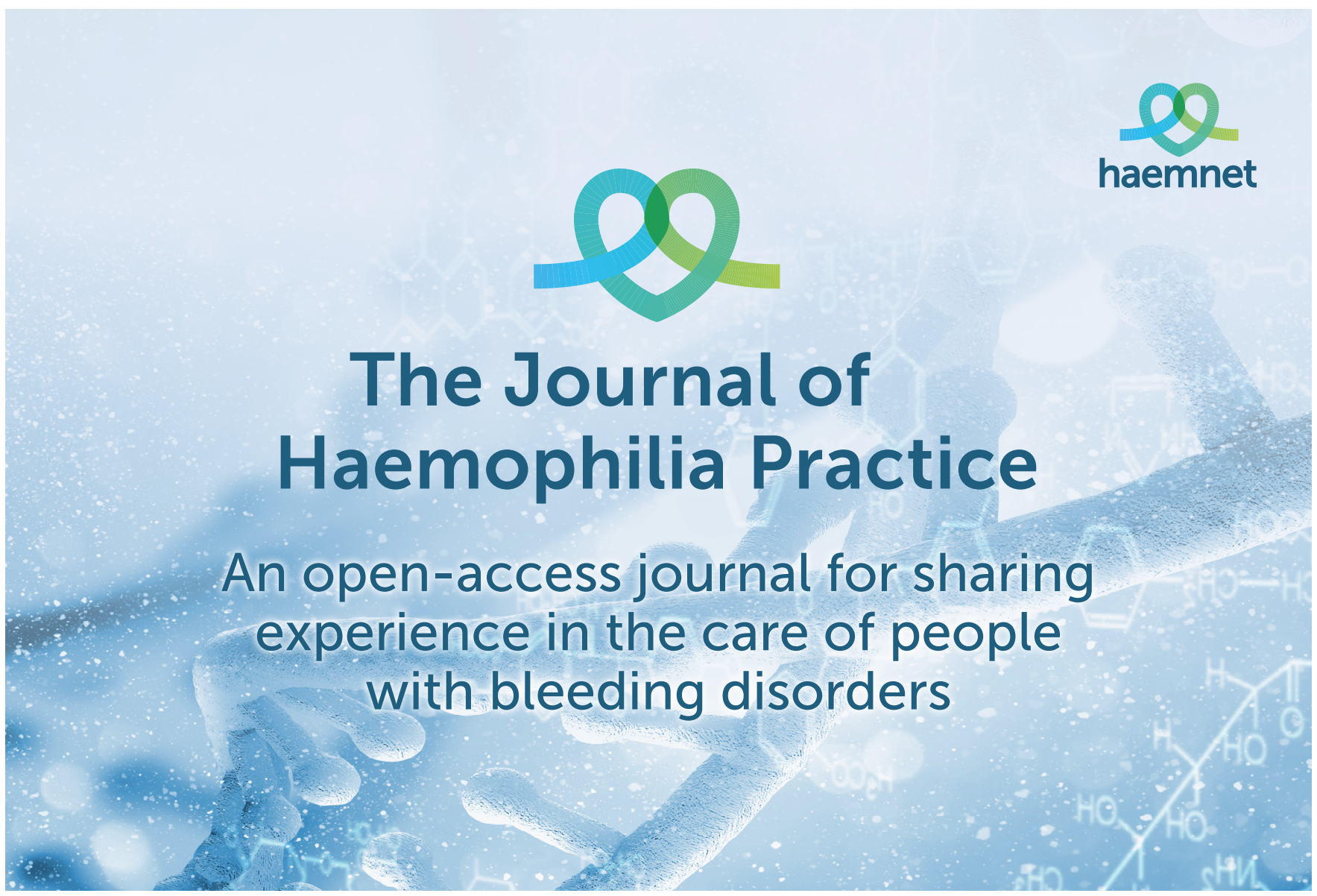


Table 1. Study characteristics

1 Jacobson $\mathrm{K}$, Hooke C. Telehealth videoconferencing for children with hemophilia and their families: a clinical project. J PEDIATR ONCOL NURS 2016; 33(4): 282-8.

\begin{tabular}{l} 
Month/year \\
\hline Country \\
\hline Study design \\
\hline Patient population (n) \\
Healthcare team \\
population \& disciplines ( $n$ ) \\
\hline Virtual medium
\end{tabular}

Virtual medium October 2015 USA

Process of virtual consultation

Cross-sectional survey

Severe haemophilia, ages $2-18(n=12)$

HTC staff $(n=4)$ : healthcare providers, HTC nurse

Telephone, videoconferencing, email with photographs

- Patient contacts clinic by phone to discuss bleeding with HTC nurse

- Videoconferencing scheduled if HCP feels visualisation would be helpful

- Documentation undertaken as a phone message

Outcomes

Patients: Easy to access, easy to set up consultation with HCPs, felt that HCPs have a better understanding of bleed using video when compared to phone calls alone, $50 \%$ felt more confident caring for child after video conference.

HCPs: $75 \%$ feel that same information could be achieved with phone call, $50 \%$ found telehealth visit to be better than phone call, $50 \%$ indifferent to video vs. phone call, $75 \%$ believe video consultations saved the need for patients to visit the hospital.

2 O'Donovan M, Buckley C, Benson J, et al. Telehealth for delivery of haemophilia comprehensive care during the COVID-19 pandemic. HAEMOPHILIA 2020; 26(4): 984-990.

\begin{tabular}{|c|c|}
\hline Month/year & September 2020 \\
\hline Country & Ireland \\
\hline Study design & Cross-sectional survey \\
\hline Patient population (n) & Ages $16-91(n=731)$ \\
\hline $\begin{array}{l}\text { Healthcare team } \\
\text { population \& disciplines ( } n)\end{array}$ & $\begin{array}{l}\text { Total } n=21 \text { : nurses }(n=9) \text {, physicians }(n=8) \text {, allied health professionals (physiotherapist, } \\
\text { psychologist, social worked; } n=3) \text {, dentist }(n=1)\end{array}$ \\
\hline Virtual medium & Telephone, videoconferencing \\
\hline $\begin{array}{l}\text { Process of virtual } \\
\text { consultation }\end{array}$ & $\begin{array}{l}\text { - Telephone consultations using speaker phones and headsets } \\
\text { - Video consultations: Blue Eye software connects patient's smartphone to HCP on a } \\
\text { web-based platform via secure SMS or email link }\end{array}$ \\
\hline Outcomes & $\begin{array}{l}\text { - Increased level of clinical activity/number of consultations } \\
\text { - } 60 \% \text { reduction in non-attendance rates } \\
\text { Patients: Improved access, reduced inconvenience, easy to use, enabled good } \\
\text { communication, no need to travel or pay for parking. Limitations included accessibility } \\
\text { issues, technical difficulties, patient preference of face-to-face interaction. } \\
\text { HCPs: } 90 \% \text { confident with telephone consultation, } 86 \% \text { confidence with video } \\
\text { consultation, } 79 \% \text { positive about teleconsultation and would continue to offer } \\
\text { teleconsultation after, } 88 \% \text { would recommend to colleagues, } 38 \% \text { and } 71 \% \text { experienced } \\
\text { telephone and video technical issues, respectively. } 67 \% \text { reported lack of adequate } \\
\text { resource to undertake video consultations. }\end{array}$ \\
\hline
\end{tabular}

3 Mondorf W, Eichler H, Fischer R, et al. Smart Medication TM, an electronic diary for surveillance of haemophilia home care and optimization of resource distribution. HAMOSTASEOLOGIE 2019; 39(4): 339-346.

\begin{tabular}{l} 
Month/year \\
\hline Country \\
\hline Study design \\
\hline Patient population $(n)$ \\
\hline Healthcare team \\
population \& disciplines ( $n$ ) \\
\hline Virtual medium
\end{tabular}

November 2019

Germany

Retrospective cohort

Haemophilia A and B, ages 24-48 ( $n=663)$

Physicians, HTC staff

Phone application involving telephone, text messages, photographs 

Process of virtual
consultation
- Direct telephone or text message
- Send photograph of bleed
- Single button message "I was admitted to hospital" during emergencies
Outcomes
- 113,530 entries for infusions administered on Smart Medication
- Reduced bleeding frequency following usage of Smart Medication

4 Zhang A, Liu W, Poon M-C, et al. Management of haemophilia patients in the COVID-19 pandemic: Experience in Wuhan and Tianjin, two differently affected cities in China. HAEMOPHILIA 2020; 26(6): 1031-1037.

\begin{tabular}{l|l}
\hline Month/year & September 2020 \\
\hline Country & China \\
\hline Study design & Cross-sectional population-based study \\
\hline Patient population $(\mathrm{n})$ & Children and adults (Wuhan, $\mathrm{n}=380 ;$;ianjin, $\mathrm{n}=429)$ \\
\hline $\begin{array}{l}\text { Healthcare team } \\
\text { population \& disciplines }(\mathrm{n})\end{array}$ & Physicians, nurses \\
\hline Virtual medium & Telephone, videoconferencing, text message with photographs \\
\hline $\begin{array}{l}\text { Process of virtual } \\
\text { consultation }\end{array}$ & $\begin{array}{l}\text { - Online clinics for video and text-picture consultation/counselling } \\
\text { - Online education classes }\end{array}$ \\
\hline Outcomes & - Reduced need for frequent in-person visits to healthcare facilities
\end{tabular}

5 Ludikhuize L, Jansen ME, Hooimeijer HL, de Bont ES, Tamminga RY. Feasibility of video consultations on case of acute complications in children with haemophilia. HAEMOPHILIA 2016; 22(6): e567-e570.

\begin{tabular}{l} 
Month/year \\
\hline Country \\
\hline Study design \\
\hline Patient population $(\mathrm{n})$ \\
\hline Healthcare team \\
population \& disciplines ( $\mathrm{n}$ \\
\hline Virtual medium \\
\hline $\begin{array}{l}\text { Process of virtual } \\
\text { consultation }\end{array}$
\end{tabular}

Outcomes
October 2016

Netherlands

Cross-sectional survey

Severe and moderate haemophilia, ages $>6$ months $(n=21)$

Paediatric haematologist, specialist nurse

Telephone, videoconferencing, photographs sent by phone

- Patient calls hospital to ask for a video consultation

- HCP generates an appointment and patient receives an email invitation with a link to a real-time video consultation that begins within 5 minutes

- $88 \%$ video consultations prevented hospital visits

- No misjudgements of bleeding episodes were encountered afterwards

Patients: Most patients/parents liked the video consultation, believed that using a webcam contributed to a better consultation, easy to use, more convenient than in-person hospital visit.

HCPs: Physicians thought using video helped judge severity of bleeding.

6 Álvarez-Román MT, De la Corte-Rodríguez H, Rodríguez-Merchán EC, et al. COVID-19 and telemedicine in haemophilia in a patient with severe haemophilia A and orthopaedic surgery. HAEMOPHILIA. 2020 Nov 3. [Epub ahead of print].

\begin{tabular}{l|l}
\hline Month/year & June 2020 \\
\hline Country & Spain \\
\hline Study design & Case report \\
\hline Patient population $(\mathrm{n})$ & Severe haemophilia A, age $56(\mathrm{n}=1)$ \\
\hline $\begin{array}{l}\text { Healthcare team } \\
\text { population \& disciplines }(\mathrm{n})\end{array}$ & Haematologists, physiatrists, orthopaedic surgeons, physiotherapists \\
\hline Virtual medium & Videoconferencing, email with photographs \\
\hline
\end{tabular}




\begin{tabular}{l|l}
$\begin{array}{l}\text { Process of virtual } \\
\text { consultation }\end{array}$ & $\begin{array}{l}\text { - Early postoperative: physicians communicated with the patient daily through } \\
\text { videoconferencing } \\
\text { - Late postoperative: patient emailed images of his knee to allow haematologists to } \\
\text { adjust treatment accordingly } \\
\text { - Daily email contact with the Haematology Department } \\
\text { - Physiotherapy sessions conducted online with the help of patient's relatives }\end{array}$ \\
\hline Outcomes & $\begin{array}{l}\text { - Patient recovered } \\
\text { - Providers found telemedicine to be useful for the Haematology Service and the } \\
\text { Orthopaedic and Rehabilitation Departments }\end{array}$ \\
\hline
\end{tabular}

HCP: Health care professional

HTC: Haemophilia treatment centre

\section{APPENDIX: Search strategies}

Database: Embase <1974 to 2020 October 23> Search Strategy:

1 telemedicine/ (26865)

2 telemedic*.mp. (33375)

3 virtual.mp. (85819)

4 remote.mp. (92061)

5 online.mp. (196046)

6 telehealth/ (7593)

7 exp telecommunication/ (75872)

8 telehealth.mp. (11717)

9 telecommunic*.mp. (27328)

10 teleconsult*.mp. (10848)

11 mobile health*.mp. (5743)

12 mhealth.mp. (4757)

13 telemonitor*.mp. (4272)

14 video conferenc*.mp. (1448)

15 videoconferenc*.mp. (5623)

16 videoconferencing/ (4529)

17 virtual visit*.mp. (327)

18 telerehab*.mp. (1439)

19 telephone visit*.mp. (155)

20 hemophilia/ (19114)

21 Hemophili*.mp. (42023)

22 hemophilia A/ (21239)

23 hemophilia B/ (7944)

24 Haemophili*.mp. (14590)

25 Christmas diseas*.mp. (133)

26 Factor VIII deficienc*.mp. (639)

29 Factor XI deficienc*.mp. (751)

30 Rosenthal syndrome.mp. (778)

31 royal disease.mp. (19)

32 blood clotting factor deficiency/ (923)

33 factor 8 deficienc*.mp. (26)

34 factor 9 deficienc*.mp. (6)

35 factor 11 deficienc*.mp. (1151)

36 factor 13 deficienc*.mp. (1037)

37 factor deficienc*.mp. (2807)

38 factor XIII deficienc*.mp. (760)

39 F VIII deficienc*.mp. (7)

40 FVIII deficienc*.mp. (363)

$41 \quad \mathrm{~F}$ IX deficienc*.mp. (3)

42 FIX deficienc*.mp. (182)

43 F XI deficienc*.mp. (19)

$44 \quad$ FXI deficienc*.mp. (467)

$45 \quad F X I I I$ deficienc*.mp. (28)

46 FXIII deficienc*.mp. (540)

471 or 2 or 3 or 4 or 5 or 6 or 7 or 8 or 9 or 10 or 11 or 12 or 13 or 14 or 15 or 16 or 17 or 18 or 19 (438724)

4820 or 21 or 22 or 23 or 24 or 25 or 26 or 27 or 28 or 29 or 30 or 31 or 32 or 33 or 34 or 35 or 36 or 37 or 38 or 39 or 40 or 41 or 42 or 43 or 44 or 45 or 46 (47986)

27 Factor IX deficienc*.mp. (357)

$49 \quad 47$ and 48 (557) 
Database: Ovid MEDLINE(R) ALL <1946 to October 23, 2020> Search Strategy:

Telemedicine/ (24455)

telemedic*.mp. (30936)

virtual.mp. (62770)

Remote Consultation/ (4886)

remote.mp. (77587)

online.mp. (127647)

teleconsult*.mp. (1351)

telemonitor*.mp. (1671)

telehealth.mp. (5862)

mhealth.mp. (5076)

mobile health.mp. (8213)

video conferenc*.mp. (840)

videoconferenc*.mp. (3161)

virtual visit*.mp. (226)

exp Telecommunications/ (94549)

Telecommunic*.mp. (8668)

exp Telerehabilitation/ (413)

telerehab*.mp. (1064)

telephone visit*.mp. (84)

Hemophilia A/ (20710)

Hemophilia B/ (4384)

Hemophili*.mp. (26582)

Haemophili*.mp. (9660)

Christmas diseas*.mp. (330)

Factor VIII deficiency.mp. (413)

Factor IX deficiency.mp. (260)
27 Factor XI Deficiency/ (764)

28 Factor XI deficiency.mp. (864)

29 Rosenthal syndrome.mp. (917)

30 royal disease.mp. (20)

31 Factor XIII Deficiency/ (660)

32 factor XIII deficienc*.mp. (814)

33 factor 8 deficienc*.mp. (260)

34 factor 9 deficienc*.mp. (2)

35 factor 11 deficienc*.mp. (1)

36 factor 13 deficienc*.mp. (10)

37 factor deficienc ${ }^{*} . \mathrm{mp} .(1210)$

38 FVIII deficienc*.mp. (151)

39 FIX deficienc*.mp. (72)

40 FXIII deficienc*.mp. (244)

$41 \quad$ FXI deficienc*.mp. (216)

$42 \quad$ F VIII deficienc*.mp. (5)

43 FIX deficienc*.mp. (1)

$44 \quad F$ XIII deficienc*.mp. (19)

$45 \quad \mathrm{FXI}$ deficienc*.mp. (6)

461 or 2 or 3 or 4 or 5 or 6 or 7 or 8 or 9 or 10 or 11 or 12 or 13 or 14 or 15 or 16 or 17 or 18 or 19 (355256)

4720 or 21 or 22 or 23 or 24 or 25 or 26 or 27 or 28 or 29 or 30 or 31 or 32 or 33 or 34 or 35 or 36 or 37 or 38 or 39 or 40 or 41 or 42 or 43 or 44 or 45 (31850)
Database: PubMed <inception to 2020 October 24> Search Strategy:

(("telemedicine"[All Fields]) OR (telemedic*) OR ("virtual"[All Fields]) OR ("remote"[All Fields]) OR ("online"[All Fields]) OR ("telehealth"[All Fields]) OR (telecommunic*) OR ("telecommunication"[All Fields]) OR (teleconsult*) OR ("teleconsultation"[All Fields]) OR (telemonitor*) OR ("telemonitor"[All Fields]) OR ("mobile health"[All Fields]) OR ("mhealth"[All Fields]) OR ("video conferenc*") OR ("video conferencing"[All Fields]) OR ("virtual visit*") OR ("virtual visit"[All Fields]) OR ("telephone visit*") OR ("telephone visit"[All Fields]) OR ("videoconference"[All Fields]) OR (videoconference*) OR (telerehab*) OR ("telerehabilitation"[All Fields])) AND ((Hemophili*) OR (“Hemophilia"[All Fields]) OR (Haemophili*) OR ("Haemophilia” [All Fields]) OR ("Christmas diseas*") OR ("Christmas Disease"[All Fields]) OR ("Factor VIII deficienc*") OR ("factor viii deficiency" [All Fields]) OR ("Factor IX deficienc*") OR ("factor ix
deficiency"[All Fields]) OR ("Factor XI deficienc*") OR ("factor xi deficiency"[All Fields]) OR ("factor XIII deficienc*") OR ("factor xiii deficiency"[All Fields]) OR ("Rosenthal syndrome") OR ("rosenthal syndrome"[All Fields]) OR ("royal disease") OR ("royal disease"[All Fields]) OR ("plasma thromboplastin component deficienc ${ }^{\star}$ ") OR ("plasma thromboplastin component deficiency"[All Fields]) OR ("factor 8 deficienc ${ }^{\star \prime}$ ) OR ("factor 8 deficiency"[All Fields]) OR ("factor 9 deficienc ${ }^{\star}$ ) OR ("factor 9 deficiency"[All Fields]) OR ("factor 11 deficienc*") OR ("factor 11 deficiency"[All Fields]) OR ("factor 13 deficienc*") OR ("factor 13 deficiency"[All Fields]) OR ("factor deficienc*") OR ("factor deficiency"[All Fields]) OR ("factor deficiency"[All Fields]) OR ("F VIII deficienc $\left.c^{\star \prime}\right)$ OR ("F IX deficienc $\left.c^{\star}\right)$ OR ("F XI deficienc $\left.c^{\star}\right)$ OR ("F XIII deficienc* $c^{*}$ ) OR ("FVIII deficienc*") OR ("FIX deficienc*") OR ("FXI deficienc $\left.{ }^{\star \prime}\right)$ OR ("FXIII deficienc ${ }^{\star}$ ")) 